\title{
Observation of rapid aerosol deposition according to AERONET data
}

\author{
Vladimir Maslov, Sabur Abdullaev*, and Bahron Nazarov \\ S. U. Umarov Physical-Technical Institute, Academy of Sciences of the Republic of Tajikistan
}

\begin{abstract}
Measurements at the AERONET station in Dushanbe showed that the diurnal variations of the aerosol optical thickness (AOT), humidity, Angström parameter depend on the stratification of the atmosphere. It is established that the dynamics of the aerosol in the ground inversion temperature layer is determined by the effect of double diffusion, which is manifested as a result of the difference in the diffusion coefficients of the components. As the temperature and aerosol concentration increase from the bottom to the top, vertical convection cells of the "salt finger" type are formed, which cause a rapid settling of dust by means of condensation of water vapor and coagulation of particles and water droplets.
\end{abstract}

\section{Introduction}

For the first time, the effect of rapid aerosol deposition was observed in Dushanbe more than 30 years ago, when during nephelometric measurements it turned out that for about two hours, in the morning and in the evening, the concentration of the surface aerosol increases dramatically. It is known that episodes of a dusty atmosphere usually end abruptly, with a short rain. Later it turned out that during the rapid deposition of the aerosol there is a sharp decrease in the surface ozone content. The deposition of particles in these periods can even be seen when illuminated by a spotlight beam. In the vertical beam of the lidar, rapid precipitation was observed at night as a downward movement at a constant speed of about $1 \mathrm{~m} / \mathrm{s}$ of luminous spots about 10 centimeters high.

It was possible to associate the observed phenomenon with the surface temperature inversion formed at the same time interval [1]. It was possible to carry out a detailed analysis after 2010 according to measurements of the solar photometer of the AERONET station. It turned out that during the surface inversion the aerosol optical thickness (AOT) and the humidity of the air sharply decrease and the particle size increases. After the end of the inversion, these parameters are restored almost to the previous level (Figs. 1,2).

\section{Variations of aerosol parameters with different air dustiness}

Measurements were carried out in 2010-2017. Data on AOT from AERONET website was used as a dustiness criterion. Usually we distinguish 3 classes of dust episodes, differing in aerosol optical thickness: weak haze, dust mist and dust storm (strong dust mist). With a low dust content of the atmosphere in the diurnal variation of the AOT, a minimum is usually observed in the middle of the day (see Figs. 1c, 2b) [1]. Such behavior is observed during normal stratification by temperature (decrease of $\mathrm{T}$ with height). This is not associated with rapid settling and is apparently the result of evaporation of droplets and water shells of particles in the first half of the day and condensation of water vapor on the particles from the surface into the second half. The daily minimum is a balance between the evaporation of droplets during solar heating and the condensation of water vapor. Authors [1] provide similar data for the daily variation of aerosol extinction in haze. The boundary between haze and mist associated with dust intrusion is very blurred, but the behavior of extinction may differ significantly. The main condition for the daily minimum of aerosol extinction is the absence of dust intrusions carrying large particles. When the dust content changes, it can be established that the diurnal variation of the AOT is related to dynamic processes in dusty air.

Figure 1 shows the variation of AOT, humidity and Ångström parameter for three days with different dust content of air at a wavelength $\lambda=1020 \mathrm{~nm}$. Figures 1a and $1 \mathrm{~b}$ show how the light haze passes into dust mist. With an increase in the degree of dustiness, not only does the AOT increase, but the daily variation of AOT also changes.

The diurnal variation of the dusty atmosphere parameters measured at AERONET station shows that the AOT, humidity and particle size are closely related to each other. In conditions of prolonged dust mist or dust haze AOT has a wide flat minimum in the middle of the day (Figs. 1c, 2b), as well as the nature of the moisture content and the Angstrom parameter. That is, the content of water vapor decreases in the first half of a day, then increases again. The corresponding maximum of the Ångström parameter means that the sizes of the particles initially decrease and then increase. Obviously, such behavior of the atmospheric parameters is determined by

\footnotetext{
* Corresponding author: sabur.f.abdullaev@gmail.com
} 

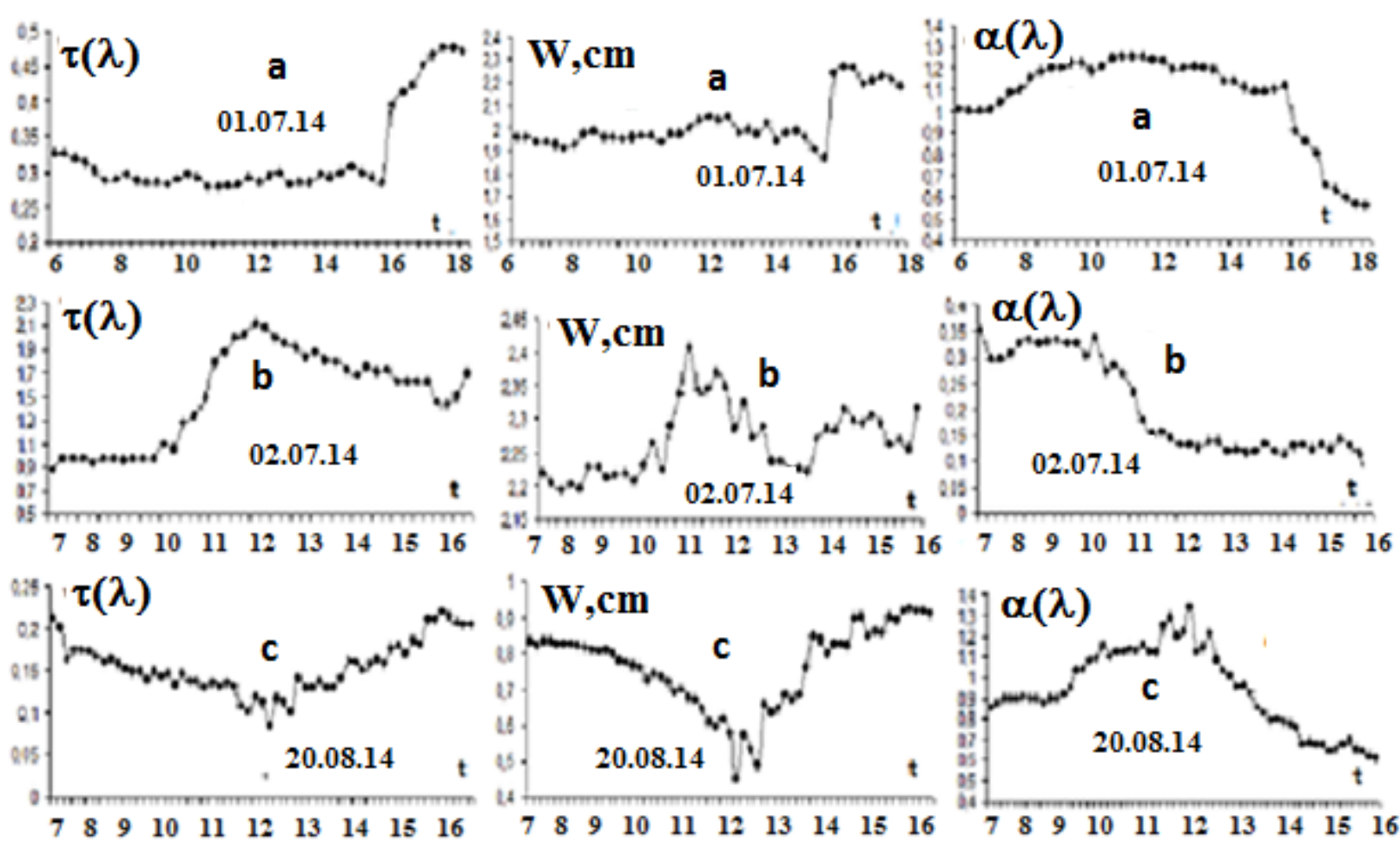

Figure 1. The day variation of AOT for $\lambda=1020 \mathrm{~nm}$, humidity and the Ångström parameter for dust storm (a), dust mist (b), a light dust haze (c).

the balance of evaporation and condensation of water vapor on droplets and aerosol particles.

After a heavy rain (Fig. 2c), a sufficiently large amount of liquid water is absent in the ground air layer, the evaporation of droplets is not realized and AOT does not decrease.

More interesting are the sharp short minima, after which their previous values are almost restored. Analysis of the physical conditions of the atmosphere during the
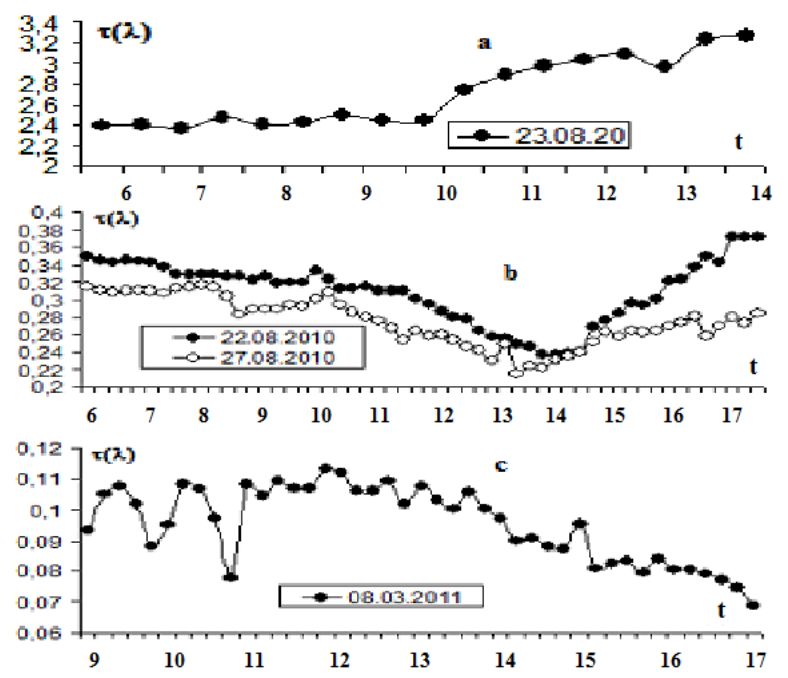

Figure 2. The diurnal variations of AOT for $\lambda=500 \mathrm{~nm}$ : dust mist (a); dust haze (b); weak dust haze, a clear day, after a torrential rain $(\mathrm{c})$. observation period made it possible to establish that such phenomena are associated with the formation and destruction of convective double-diffusive cells in the layer of temperature inversion (near the ground or at height).

In dust mist, the maximum of AOT corresponds to the largest dust content (Figs. 1b, 2a). The evening minimum of AOT can be unambiguously associated with the enhancement of particle deposition. In summer, at a high air temperature and a low average daily AOT, almost the same diurnal course is observed (Fig. 1b), as for background dust haze (Fig. 2b), only a minimum is slightly split. The growth of AOT can be related either to aerosol removal from the ground surface during the convection of heated air, or to the condensation of water vapor.

\section{The effect of double diffusion in the atmosphere}

Analysis of the physical parameters of the atmosphere made it possible to establish the cause of the observed phenomena [2-4]. It was shown that in a stratified dusty atmosphere, the effects of double diffusion can appear [5]. Double diffusion is Rayleigh-Bénard type convection in two-phase systems, when the gradients of two physical parameters give opposite contributions to the density of the system. Convective transfer of heat and impurities included under the condition for the effective Rayleigh number: 


$$
R a=\left(L e \cdot R a_{s}-R a_{T}\right) \geq \frac{27}{4} \pi^{4}=657 .
$$

Here $R a_{\mathrm{T}}$ is the Rayleigh number for thermal diffusion, $R a_{\text {s }}$ the Rayleigh concentration number, the kinematic viscosity of air, $D_{\mathrm{T}}, D_{\mathrm{S}}$ - the diffusion coefficients of temperature and concentration, $L e=D_{\mathrm{T}} / D_{\mathrm{S}}$ - the inverse Lewis number.

Conditions for the effect of "salt fingers" in an aero disperse medium are realized with a stable stratification of the atmosphere with respect to temperature, i.e. in the layer of surface inversion. At the same time, the impurity concentration must also increase from the bottom up. In this case, a rapid precipitation of the aerosol occurs, with the formation of narrow long convective cells.

If $R_{\rho}=\beta_{T} T_{z} / \beta_{S} S_{z}$, the ratio of contributions to the density of the medium, from parameters with stable and unstable stratification (where $T_{\mathrm{z}}$ and $S_{\mathrm{z}}$ are the temperature and concentration gradients) then the condition for the convective regime of "salt fingers" is

$$
T_{z}>0 ; S_{z}>0 ; 1<R_{\rho}<D_{T} / D_{S} .
$$

Let us estimate the parameters of double diffusion for dust haze with mass concentration $\mathrm{C}_{\mathrm{m}}=0.5 \mathrm{mg} / \mathrm{m}^{3}$ and particle density $\rho_{\text {part }} \approx 2.5 \mathrm{~g} / \mathrm{cm}^{3}$. The largest temperature lapse rate in the ground inversion layer according to meteorological data is $\partial T / \partial z \approx 7.5 \cdot 10^{-4} \mathrm{~K} / \mathrm{cm}$ [6]. Even at $\mathrm{h}=1 \mathrm{~cm}$, the effective Rayleigh number of larger particles exceeds the critical value, i.e. convective instability arises. The diffusion coefficient of particles, the Rayleigh number, the rate of gravitational sedimentation (terminal fall speed) $V_{g}$, and the velocity of Brownian motion $\left\langle V_{B}^{2}\right\rangle^{1 / 2}$ are given in Tab. 1 .

The larger the gradient of the particle concentration, the larger the flow of double diffusive convection, the faster the particles deposition. The larger the particle size, the higher the temperature gradient, double diffusive settling can occur. Due to the higher diffusion coefficient, the submicron fraction of the particles is also involved in convective deposition caused by larger particles, and coagulation of particles is enhanced in a denser flow at the center of the convective cell [5]. Such a mechanism explains the decrease in the fraction of fine particles of particles during dust intrusions. As the dust haze develops and AOT grows (Figs. 1a, 1b), the Ångström parameter decreases, that is, the particle size of the aerosol increases. In the case of dust haze, the Ångström parameter changes almost synchronously with AOT (Fig. 1c), that is, the increase in particle size in the first half of the day may be due to coagulation or condensation of water vapor. In the second half of the day, the growth of the Ångström parameter and the decrease in particle size are most likely due to the evaporation of water droplets.

In convective double-diffusive cells, a sharp increase in the size of droplets and aerosol particles takes place, which is associated with the condensation of water vapor and with coagulation of particles.

At the same time, an increase in particle size leads to their rapid deposition, since the rate of gravitational deposition of $V_{g}$ (see Tab. 1) increases sharply.

The effect of rapid deposition of aerosol continues for some time after the termination of the doublediffusive conditions of convective instability, until large particles fall or lose their water shell. In the case of elevated temperature inversions, the effect of rapid deposition also occurs, but due to the relatively small thickness of such an inversion layer, it gives a background contribution to aerosol extinction and, reflecting down aerosol particles, often serves as the upper boundary of the dusty layer.

The minima in the diurnal variation of AOT (Fig. 1,2) are evidently due to the coagulation of aerosol particles and droplets, or by the evaporation of droplets. When the particle size distribution changes, and the number of larger particles increases, due to the strong absorption of light by large particles $(2 \pi \mathrm{D} \kappa / \lambda>>1)$. Here $\kappa$ is imaginary part of refractive index. With larger aerosol particles of dust mist, the gentle daytime minimum of AOT is due to a decrease in the attenuation of light by the particles, since the massive particles attenuate the light less than the same particles separately.

Water vapor, condensing on the droplets and aerosol particles, provides their integration by a more efficient coalescence mechanism, which is characteristic for water droplets and ensures the growth of raindrops and fog [7]. This is confirmed by the frequent completion of dust episodes with a short rain, even in the driest summer period. The daily course of humidity, basically, repeats the course of AOT. Reduction of humidity in the middle of the day in conditions of background dustiness (dust haze) is associated with condensation of water vapor in the cooling air in descending convective cells (Fig. 1c).

Water droplets and watered particles settling take

Table 1. The parameters of double diffusion in the atmosphere with the largest temperature gradient in ground temperature inversion for particles of different diameters.

\begin{tabular}{|l|l|l|c|c|c|}
\hline$d, \mu \mathrm{m}$ & $D_{s}, \mathrm{~cm}^{2} / \mathrm{s}$ & $V_{g}, \mathrm{~cm} / \mathrm{s}$ & $\left\langle V_{B}^{2}\right\rangle^{0.5}, \mathrm{~cm} / \mathrm{s}$ & $R a_{T}$ & $R a_{S} \cdot L e$ \\
\hline 10 & $2.410^{-8}$ & 0.7 & 0.017 & 76083.5 & 120932 \\
\hline 1 & $2.7610^{-7}$ & $8.110^{-3}$ & 0.55 & 76083.5 & 11107.3 \\
\hline 0.1 & $7.0110^{-6}$ & $210^{-4}$ & 17.4 & 76083.5 & 437.3 \\
\hline 0.01 & $5.510^{-4}$ & $1.510^{-5}$ & 549 & 76083.5 & 5.6 \\
\hline
\end{tabular}


away the aerosol and water vapor from the atmosphere. When the air warms up, the ground temperature inversion disappears and the convection cells decay, the condensed water begins to evaporate, and the humidity starts to increase again. In the course of dust episodes, another condition of double diffusion may be violated: the vertical gradient of dust concentration disappears. When the double diffusion is suppressed, the daily minima of the AOT and the humidity disappear.

\section{Interpretation of observed data}

The advective flow bringing dust from southern Tajikistan to Dushanbe has a higher temperature and rolls from above, which can create temperature inversion and can lead to convective dust sedimentation.

While the wide daily minimum of AOT is a balance between the evaporation of droplets during solar heating and the condensation of evaporated water vapor, in the absence of external sources and sinks (anthropogenic and natural) and changes in solar air heating, particle size changes can occur only due to the heterogeneity of the medium, that is, the presence of stratification. Radiation cooling becomes significant only on a higher time scale, apparently more than 6 hours. The antigreenhouse effect, which leads to a stronger heating of aerosol particles in higher layers of dusty air, as the air warms up, also leads to inverse stratification and rapid dust deposition.

The change in particle size can also occur due to the effect of recondensation of water vapor (in Physics of atmosphere: Wegener-Bergeron-Findeisen process or "cold-rain process") [7]. This is the main mechanism of growth of ice particles and snowflakes, but the effect is also observed at higher temperatures Due to the dependence of the pressure of water vapor on the particle size, there is an increase in the condensation of water vapor on particles with sizes more critical. With a decrease in air temperature, this effect increases as the pressure of saturated water vapor decreases. As a result of this effect, droplets and pieces of ice in convective cells become larger and are deposited faster.

This effect makes it difficult to measure the absorption of dispersed substances by the immersion method. The effective absorption of a substance in a dispersed state is always less than in the solid phase [8].

A more complex structure of rapid deposition of aerosol associated with double-diffusive convection can be observed when measuring the surface concentration of aerosol and soot (black carbon).

In Tomsk, the surface content of aerosol and black carbon was measured, both in summer and in winter [9]. These results fully confirm the proposed model. In particular, the presence of double maxima in the morning and evening suggests that rapid deposition stops at the highest inversion level (maximum temperature difference), and then starts again as the inversion breaks down. Hourly averaging eliminates the influence of inter-day (synoptic) variability and random factors.

In summertime, both double maxima (in the morning and in the evening) appear for both the aerosol and black carbon. In summer, the first morning maximum (the soil and the air cool more slowly) and the second evening maximum (the inversion decays more slowly) are more powerful. In winter time, on the contrary, the second morning maximum (inversion slowly warms up) and evening (inversion, apparently, weak and long) are more powerful. The shift to a later time of the consequences of the powerful morning inversion and the formation of a wide daily minimum of AOT and the concentration of ground-level aerosol are due precisely to the inertness of rather large particles. These particles, descending into colder layers of air, are coated with a layer of condensed water vapor and coagulate with water droplets in the convective cells formed at the upper boundary of the inversion layer.

The mechanism of double diffusion allows us to evaluate the parameters of dynamic processes in atmosphere and monitor them, for example, according to AERONET. The described model of dynamic processes in aero disperse systems is fully applicable to natural liquid-drop systems stratified by the temperature and concentration of water droplets. Apparently, most of the short-term fluctuations in the parameters of the atmosphere, not associated with the advection of dusty air and with ground-based emissions of pollution, are due to condensation of water vapor and convective sedimentation of water droplets and aerosol particles.

The creation of the theory of condensation-induced atmospheric dynamics [10] in the last two decades has shown that water transformation, its transformation plays a key role in atmospheric processes. The doublediffusive model of convective processes in the atmosphere shows how these phenomena are realized at the microphysical level.

The studies were carried out with the financial support of the (ISTC) Projects T-1688 and T-2076.

\section{References}

1. Y.A. Pkhalagov, V.N. Uzhegov, N.N. Shchelkanov, Atmos. and Ocean. Opt. 9, 598 [in Rus.] (1996)

2. V.A. Maslov, B.I. Nazarov, S.F. Abdullaev, Rep. Acad. Sci. Tajik. 50, 241 [in Rus.] (2007)

3. V.A. Maslov, B.I. Nazarov, S.F. Abdullaev, Rep. Acad. Sci. Tajik. 60, 515 [in Rus.] (2017)

4. V.A. Maslov, B.I. Nazarov, S.F. Abdullaev, Rep. Acad. Sci. Tajik. 61, 159 [in Rus.] (2018)

5. T. Radko, Double-diffusive convection, Cambridge University Press, New York (2013)

6. P.A. Bokova, B.U. Mahmadaliev, A short course on general and synoptic meteorology (taking into account the orographic features of Tajikistan, Irfon, Dushanbe, [in Rus.] (2009)

7. R.G. Fleegle, J.A. Businger, Atmospheric Physics, Academic Press, New York (1980)

8. P.K. Khripunov, V.A. Maslov, T.I. Danilova, Atmos. and Ocean. Opt. 4, 510 [in Rus.] (1991) 
9. V. S. Kozlov, M. V. Panchenko, E. P. Yausheva Atmos. and Ocean. Opt. 1, 30 (2011)

10. A.M. Makarieva, V.G. Gorshkov, D. Sheil, A.D. Nobre, B.-L. Li, Atmos. Chem. Phys. 13, 1039-1056 (2013) 